\title{
Hierarchically Structured Iron-Doped Silver (Ag-Fe) Lotus Flowers for Efficient Oxygen Reduction Reaction
}

\author{
Gumaa A. El-Nagar*a,b, Iver Lauermann ${ }^{\text {c }}$, Radwan M. Sarhan ${ }^{\mathrm{a}, \mathrm{d}}$, and Christina Roth*b \\ a Chemistry Department, Faculty of Science, Cairo University, Giza, 12613, Egypt \\ b Institut für Chemie und Biochemie Physikalische und Theoretische Chemie, Freie Universität Berlin, 14195, Berlin, Germany \\ c Helmholtz-Zentrum Berlin für Materialien und Energie, Hahn-Meitner-Platz 1, 14109, Berlin, Germany \\ d Institute of Physics and Astronomy, University of Potsdam, Karl-Liebknecht-Strasse 24-25, 14476, Potsdam, Germany
}

\section{Abstract}

Developing cheap and efficient electrocatalysts for the oxygen reduction reaction (ORR) is vital for the immediate commercialization of fuel cells which are still limited by the high cost and low performance of the utilized commercial Pt-based electrodes. As a promising alternative, this study reports on the synthesis of hierarchical iron-doped silver lotus flowers $\left(\mathrm{AgFe}_{\text {lotus }}\right)$ by a facile chemical procedure as robust and efficient ORR electrocatalysts. Succinic acid was used as structure directing agent to tune the morphology of undoped and iron-doped silver particles. In the absence of succinic acid, ball-like silver particles were obtained, while using $2 \mathrm{mM}$ succinic acid led to peony-like flower structures. The doping of silver peony-flowers with iron resulted in lotus-like flower structures with high electrocatalytic activity for ORR together with outstanding tolerance against poisoning with various hydrocarbon impurities (HCs), in-situ generated during fuel cell operation, as well as different fuels from anodic crossover. $\mathrm{AgFe}_{\text {lotus }}$ exhibited a superior ORR activity with more than 40 times higher stability than the commercial Pt/C catalyst in alkaline media. This substantial performance enhancement is attributed to the unique lotus-like flower structures providing more electroactive surface sites, in addition to the iron dopants which are facilitating ORR charge transfer.

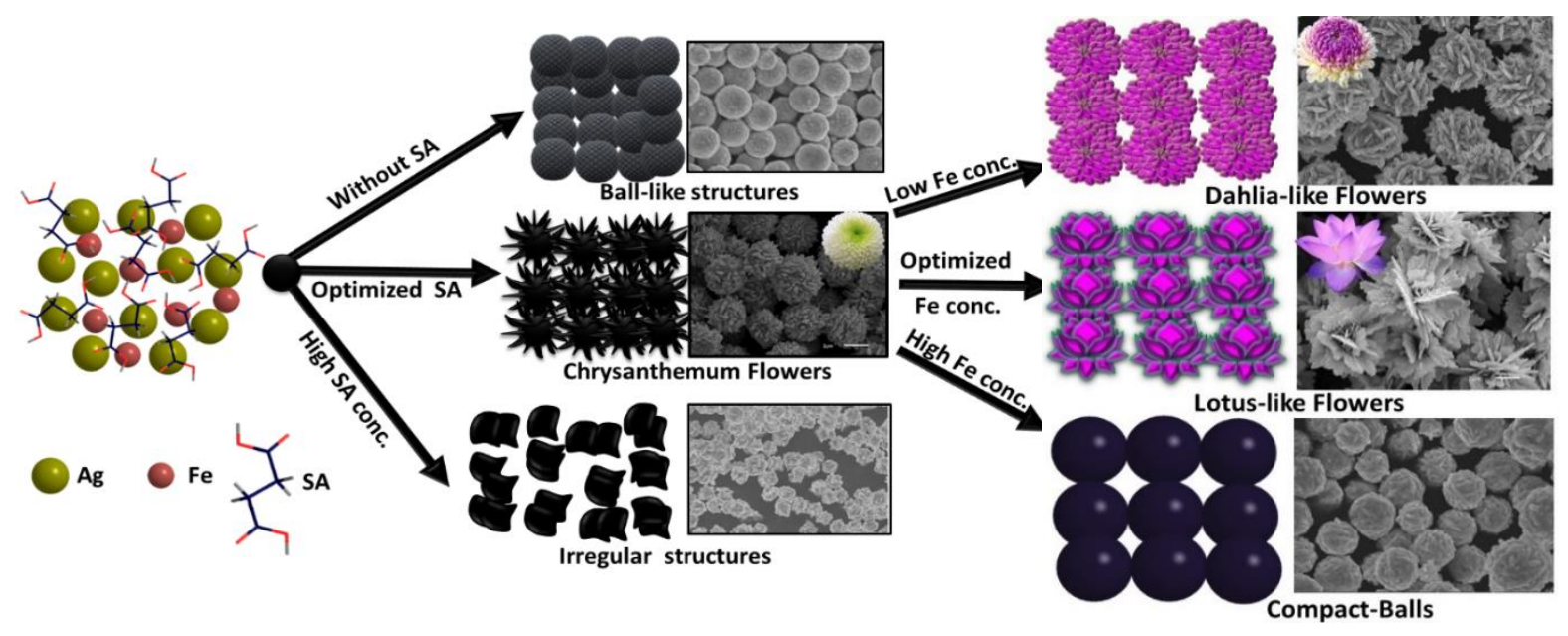

Keywords: Fuel Cells, ORR, Flower structures, silver nanostructures

*corresponding author: email address: Gumaa.elnagar@fu-berlin.de (Dr. Gumaa A. El-Nagar). 


\section{Introduction}

Development of efficient catalysts for the sluggish oxygen reduction reaction (ORR) plays a pivotal role in the overall performance and cost of polymer electrolyte membrane fuel cells (PEMFCs), which are considered as an auspicious alternative to traditional technologies based on fossil resources. However, their commercialization is obstructed by high cost and poor performance of their commercial Pt-based electrodes 1-11. The major performance losses in PEMFC originate from Pt particles agglomeration and low tolerance of Pt towards various organic and inorganic contaminants produced in-situ from degradation of various PEMFC components, as well as the platinum's poor tolerance against different anode impurities which cross-over from the anode through the membrane and result in a significant reduction of the PEMFC efficiency 9, 10, 12-14. Consequently, cheap and stable alternatives to such costly Pt-based electrodes are required for the further development of fuel cell technologies.

Alkaline fuel cells received significant attention due to alkaline media offering unique features, such as faster electrode kinetics (e.g., ORR), the use of cheap, non-precious metal electrocatalysts, reduced anodic fuel cross-over and improved water management (i.e., avoiding the possibility of water-flooding $)^{15-17}$. Made also possible by the recent advent of stable alkaline membranes. On the other hand, the silver based electrodes excellent activity and high stability under alkaline conditions make them a good ORR electrocatalyst for alkaline fuel cell applications. The high performance of silver based electrocatalysts for ORR in alkaline medium have been driven by the $\mathrm{Ag} / \mathrm{Ag}_{2} \mathrm{O}$ equilibrium potential (1.17 vs RHE) which is just below the $\mathrm{O}_{2} / \mathrm{H}_{2} \mathrm{O}$ equilibrium potential. Consequently, silver keeps its metallic state ensuring suitable conductivity of the active sites. Besides, silver is able to completely reduce oxygen into water via a 4-electron pathway within the whole ORR potential window. This is also based on the fact that silver is an exceptional hydrogen peroxide reduction electrocatalyst ${ }^{12,18-21}$.

Herein, the synthesis of 3D iron-doped silver ( $\mathrm{Ag}-\mathrm{Fe})$ hierarchically structured microflowers for efficient ORR with excellent stability and poisoning tolerance is reported. Ag-Fe flowers have been prepared via a facile and cost-effective chemical reduction method, wherein succinic acid (i.e., capping agent) was used to control the obtained Ag-Fe morphology and roughness. 


\section{Experimental}

Hierarchical silver microstructures were synthesized by a simple chemical procedure ${ }^{12}$, in which succinic acid and ascorbic acid were used as capping and reducing agents, respectively. Briefly, $1 \mathrm{ml}$ of $1 \mathrm{M} \mathrm{AgNO}_{3}$ aqueous solution was mixed with different volumes of $0.25 \mathrm{M}$ succinic acid in $15 \mathrm{ml}$ of deionized water for $10 \mathrm{~min}$ in an ice-water bath. Next, $1 \mathrm{ml}$ of $1 \mathrm{M}$ ascorbic acid was rapidly added and vigorously stirred for $25 \mathrm{~min}$. The color quickly turned to a grey color with the formation of silver particles. The same procedure was used to prepare silver-iron flowers with different iron contents. This was achieved by adding various iron concentrations to the above reaction mixtures. The microparticles were finally precipitated and washed several times with deionized water before being used. The detailed experimental information about the used chemicals, apparatus and electrode preparations are mentioned in the supplementary information.

\section{Results and Discussions}

\subsection{Morphology and Crystal Phase Control}

The crystal structure and morphology of the as-synthesized silver structures were examined first by X-ray diffraction (XRD) and scanning electron microscopy (SEM) to correlate the different synthesis parameters (e.g., capping agent and iron concentrations) to the obtained silver particles morphology.

Capping agent concentration effect: silver particles were first prepared in the absence and presence of various succinic acid (as structure directing agent) concentrations (0-3 mM). Figure 1A-F displays the corresponding SEM images. SEM images obviously reveal that the capping agent concentration has a substantial effect on the created silver particles morphology: in the absence of succinic acid (capping agents), silver particles with ball-like structures ( $\left.\mathrm{Ag}_{\text {ball }}\right)$ were formed with estimated average particle size of ca. $100 \mathrm{~nm}$ as calculated using Scherrer's formula ${ }^{12}$ (Fig. 1A). The introduction of $0.4 \mathrm{mM}$ succinic acid (SA) into the reaction mixture resulted in a creation of small buds on these ball-like silver particles (i.e, spiky ball-like particles are formed, Fig. 1B). Remarkably, those buds grow in size with the further increase of SA concentration (1 mM) leading to construction of well-assembled chrysanthemum-like flower structures (with estimated average particle size of $\sim 70 \mathrm{~nm}$, Fig. 1C). 

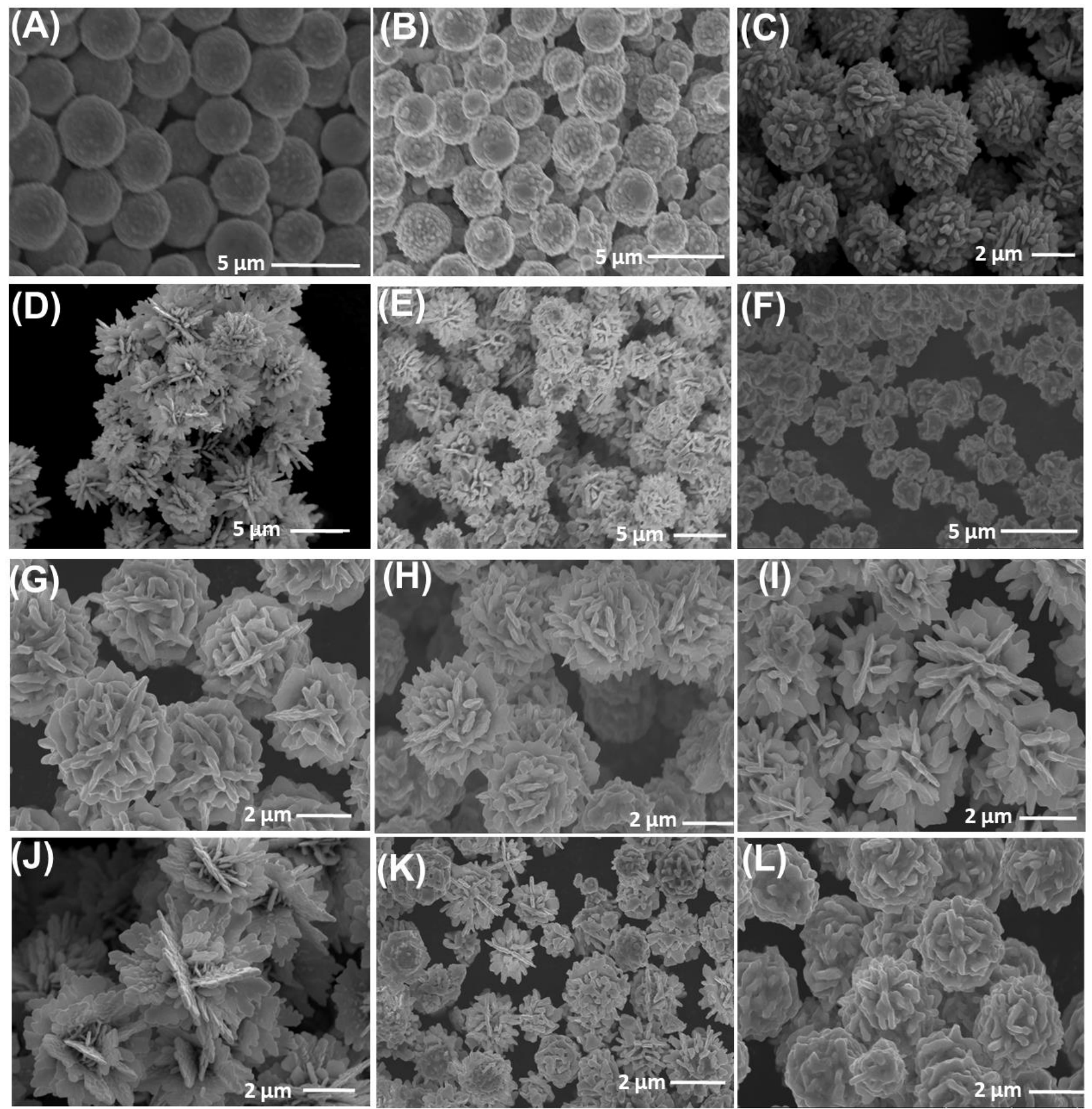

Figure 1. (A-F) SEM of silver particles created using various SA concentrations (typically 0, 0.4, 1, 2, 2.4 and $3 \mathrm{mM} \mathrm{SA}$, images A, B, C, D, E and F, respectively) and (G-L) SEM of Ag-Fe microstructures prepared by using $1 \mathrm{mM} \mathrm{SA}$ and different iron concentrations (typically, 0.04, 0.1, 0.2, 0.3, 0.4 and $0.6 \mathrm{mM}$ iron, images G, H, I, J, K and L, respectively). 
Furthermore, using 2 mM SA produced peony-like flowers ( $\mathrm{Ag}_{\text {peony }}$ ) composed of intersected nanoflakes with average particle size of $\sim 60 \mathrm{~nm}$ (Fig. 1D) with 8 times higher electrochemically active surface area compared to that of the created ball-like silver particles (see Fig. S1). Note that the further increase of SA concentration $(>2 \mathrm{mM}$ ) led to the deterioration of the flower structures (Fig. 1E\&F) concurrently with a significant decrease of the electroactive surface area and an increase of the average particle size (Fig. S1). Table S1 lists the variation of the estimated average particle sizes of the created undoped and iron doped silver particles with the capping agent and iron particles concentrations. Note that silver peony-flowers, which were created by 2 mM SA (assigned as $A g_{\text {peony }}$ ), exhibited the best performance for ORR which we attributed to their highest electrochemically active surface area (Fig. S1). Thus, we used this system to study the effect of small additions of Fe salt into the reaction mixture on their morphology and performance for ORR.

Iron doping effect: Fig. 1G-L displays the effect of Fe additions on the created silver peony-flowers morphology. As clearly seen in these SEM images, using $0.04 \mathrm{mM}$ (Fig. 1G) and $0.1 \mathrm{mM}$ (Fig. 1H) iron led to the formation of iron-doped silver $(\mathrm{Ag}-\mathrm{Fe})$ dahlia-flower structures, while $0.2 \mathrm{mM}$ and $0.3 \mathrm{mM}$ iron exhibited Ag-Fe lotus-flower structures with average particle size of $\sim 60 \mathrm{~nm}$ (Fig. 1 I\&J). However, using higher iron concentrations $(>0.3 \mathrm{mM})$ the delicate flower-like structures were damaged (Fig. $1 \mathrm{~K} \& \mathrm{~L})$ and exposed lower electrochemically active surface areas (Fig. S1).

All the above-prepared silver-based structures created using different SA and/or iron concentrations were further examined by XRD (Fig. 2A and Fig.S2). All of them exhibited the diffraction peaks characteristic of the silver face centered cubic structure ${ }^{22-24}$. Noteworthy, the intensity ratio between the $\mathrm{Ag}(111)$ and $\mathrm{Ag}$ (220) diffraction peaks significantly increased from 3.8 for the silver particles synthesized without using capping agent (Agball) to 5.2 for silver flowers created using SA as structure directing agent (Fig. 2A) ${ }^{22-24}$. As shown in Table S1, the (111)/(220) ratio increases with the capping agent concentration reaching its maximum at $2 \mathrm{mM}$. Beyond this concentration, this ratio decreases again. 

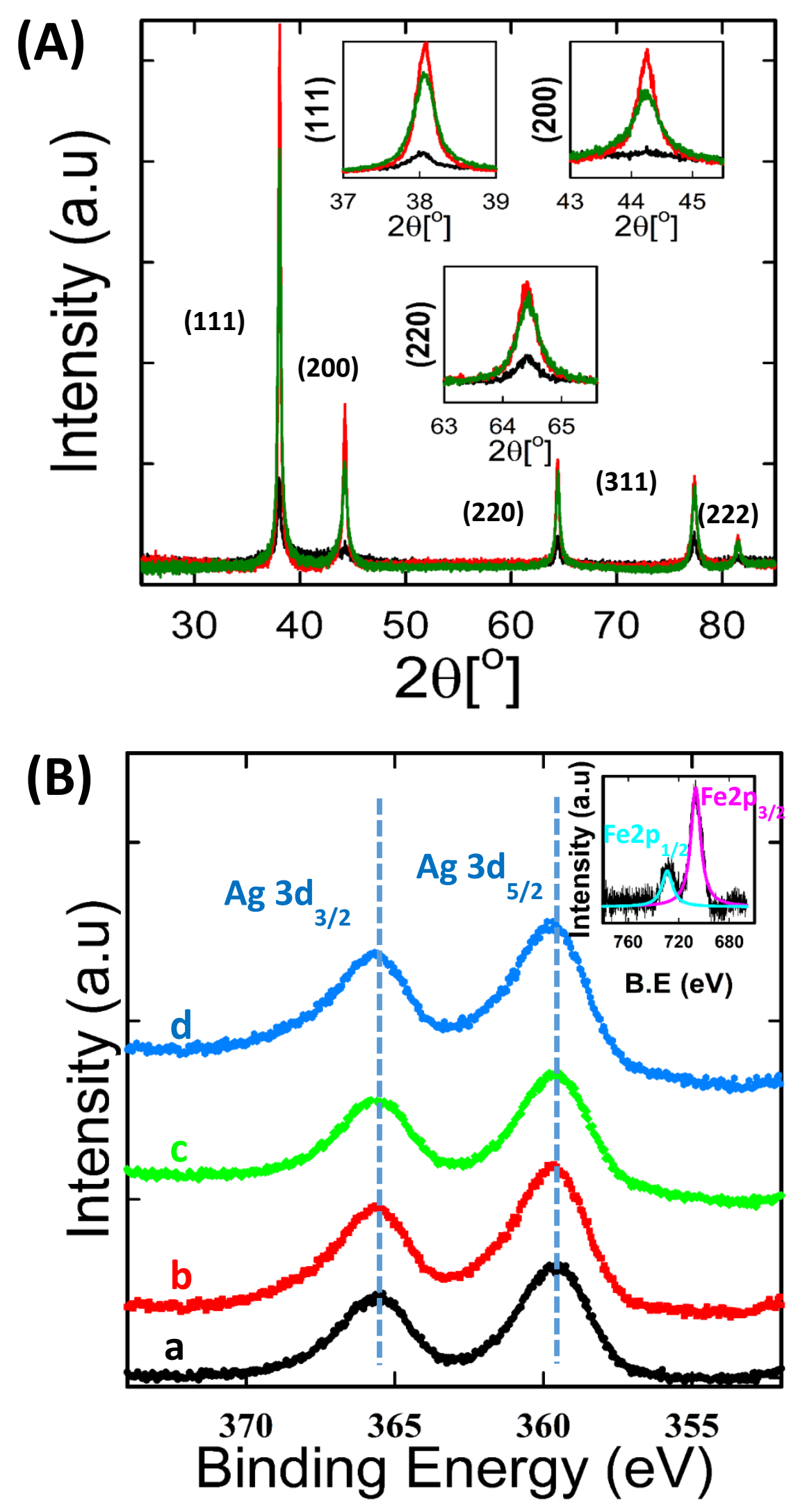

Figure 2. (A) XRD patterns of $\mathrm{Ag}_{\text {ball }}$ (black-line, prepared without SA), $\mathrm{Ag}_{\text {peony }}$ (prepared using $2 \mathrm{mM} \mathrm{SA}$, red line) and $\mathrm{AgFe}_{\text {Lotus }}$ (created using $2 \mathrm{mM} \mathrm{SA}$ and $0.2 \mathrm{mM} \mathrm{Fe}$, green line). (B) XPS high resolution spectra of $\mathrm{Ag} 3 \mathrm{~d}$ of (a) $\mathrm{Ag}_{\text {ball }}$, (b) $\mathrm{Ag}_{\text {peony }}$, (c) $\mathrm{AgFe}_{\text {dahlia }}\left(2 \% \mathrm{Fe}\right.$ ) and (d) $\mathrm{AgFe}_{\text {lotus }}$ (4\% Fe). Inset of Figure 2B shows the XPS high resolution $\mathrm{Fe} 2 \mathrm{p}$ peak of $\mathrm{AgFe}_{\text {lotus }}(4 \% \mathrm{Fe})$. 
This suggests that the (111) facet is the predominant crystal facet of the created hierarchical silver flowers and crystal growth occurs preferentially oriented parallel to the (111) plane.

We postulate the following mechanism for the formation of hierarchical silver flower-structures: (i) silver ions $\left(\mathrm{Ag}^{+}\right)$firstly become reduced by ascorbic acid to primary silver particles, (ii) these primary silver nanoparticles agglomerate and (iii) grow anisotropically into flower-like structures derived by the selective adsorption of SA on the silver facets. SA carboxylic functionalities will get adsorbed on different silver facets probably with weakest binding on the (111) crystal plane ${ }^{25-27}$. This initiates the anisotropic growth of silver spikes along the (111) direction, and those spikes develop to nanosheets with the increase of SA concentration resulting in the formation of well-assembled silver flowers ${ }^{25-27}$.

Moreover, the as-prepared silver structures composition was further determined by energy dispersive Xray spectroscopy (EDS, Fig. S3) and X-ray photoelectron spectroscopy (XPS, Fig. 2B) techniques. While EDS analysis verified the successful and homogenous decoration of the created silver flower-structures with iron (Fig. S3), the XPS measurements indicate some electronic interaction between the capping agent (SA), iron dopants and silver particles as evidenced from the slight positive shift of the Ag3d binding energy of the $\mathrm{Ag}_{\text {peony }}$ and $\mathrm{AgFe} \mathrm{e}_{\text {lotus }}$ compared to that of $\mathrm{Ag}_{\text {ball }}$ (Fig. 2B).

\subsection{Electrocatalytic Performance for ORR}

The electrocatalytic activity of $\mathrm{Ag}_{\text {ball }}, \mathrm{Ag}_{\text {peony }}$ and $\mathrm{AgFe}_{\text {lotus }}$ flowers for ORR were evaluated by recording their respective linear sweep voltammetry (LSVs) on a rotating-disk electrode (RDE) in $\mathrm{O}_{2}$-saturated $0.1 \mathrm{M} \mathrm{KOH}$.

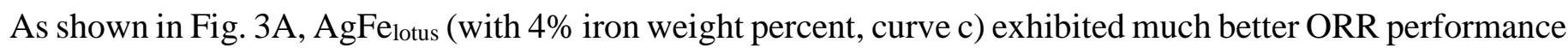
compared to $\mathrm{Ag}_{\text {ball }}$ (curve a) and $\mathrm{Ag}_{\text {peony }}$ (curve b) as demonstrated by its more positive onset potential and higher ORR current. Besides, $\mathrm{AgFe}_{\text {lotus }}$ showed a comparable catalytic activity to the commercial carbonsupported Pt electrode (curve e). It is worth to mention that higher iron weight percentages $(>4 \%)$ reduced the structures' activity for ORR significantly (curve d), an effect that we attributed to the destruction of their unique lotus-flower structures at higher iron contents as revealed in Fig. 1L. 
(A)

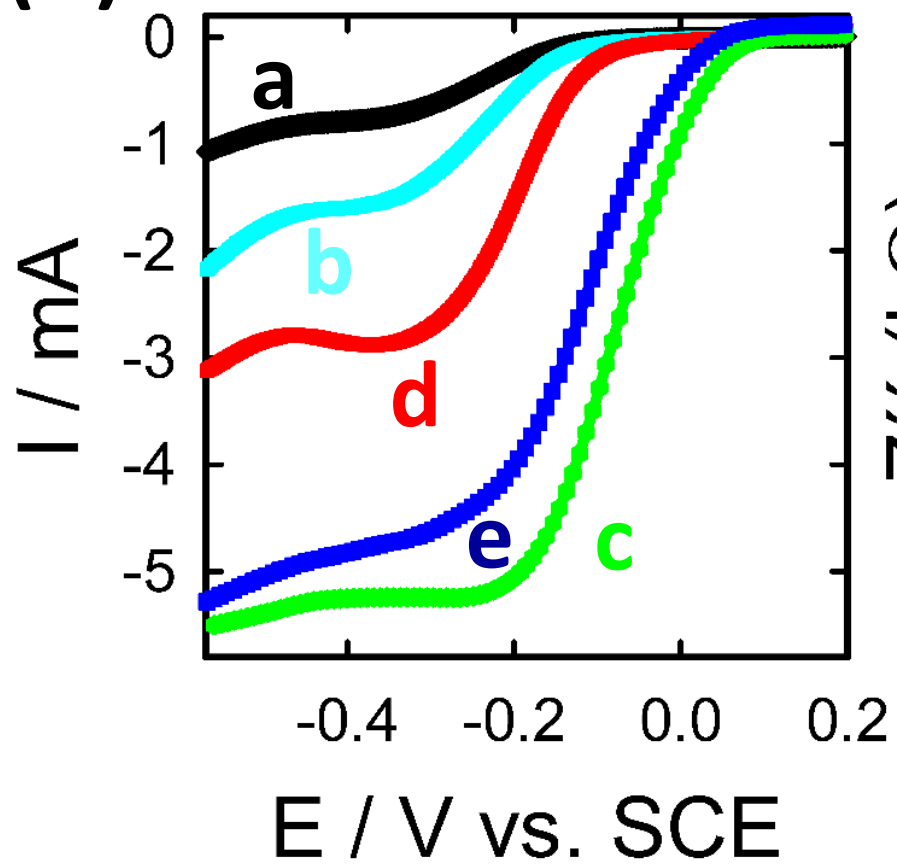

(B)

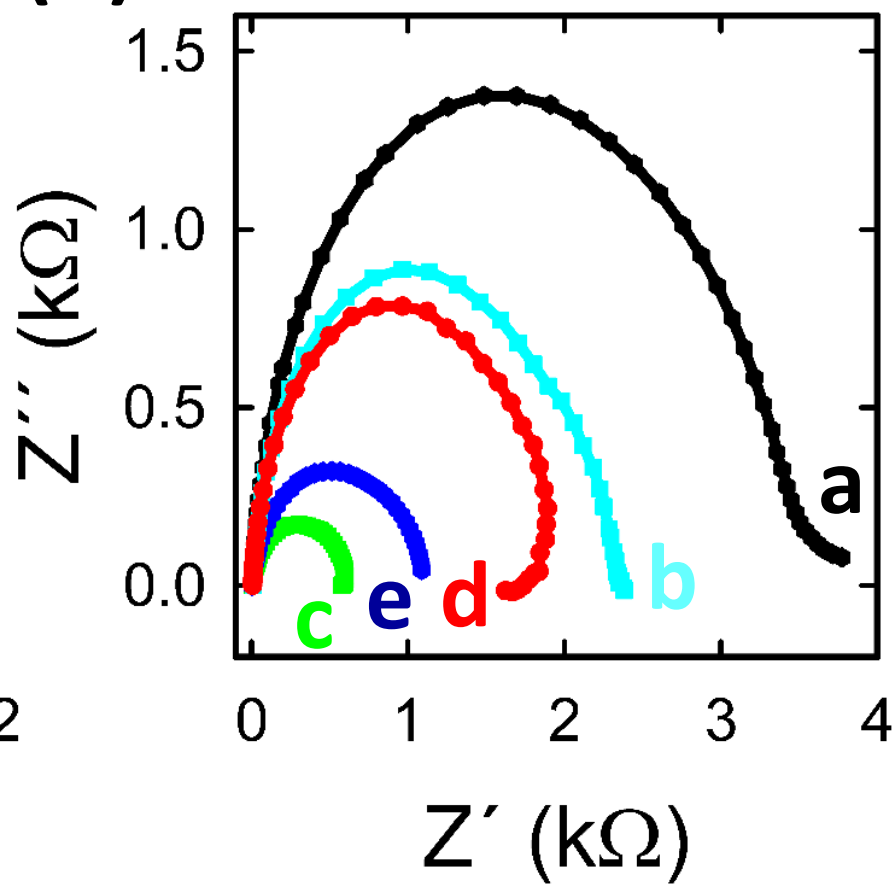

(D)

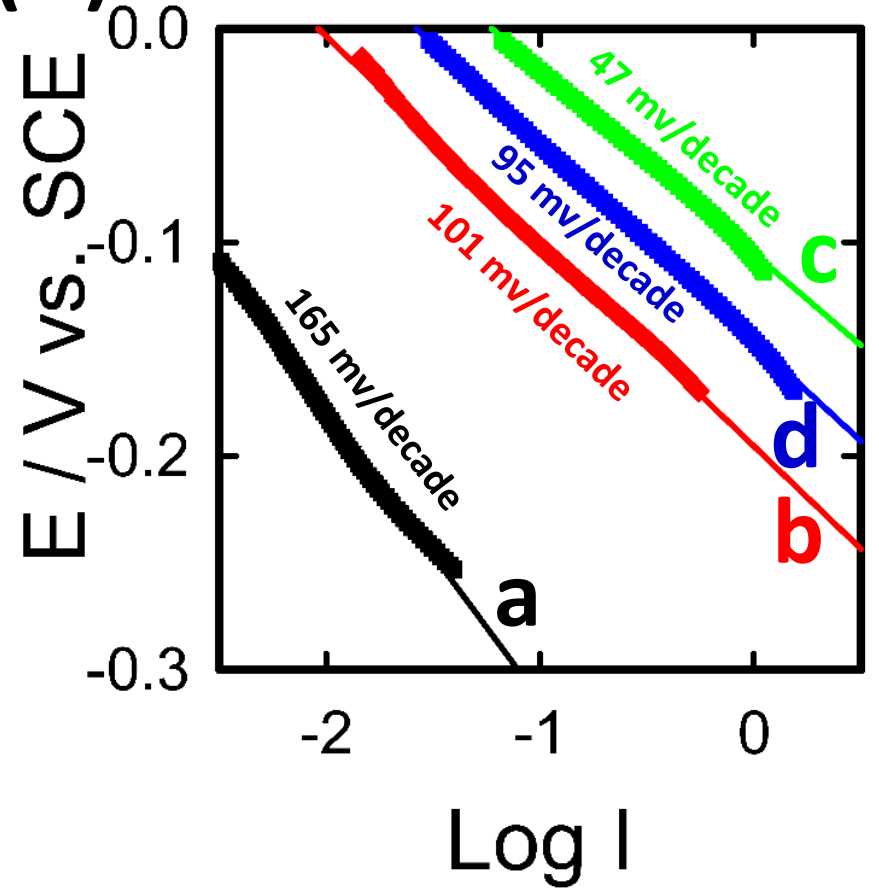

Figure 3. (A) RDE measurements obtained at (a)Agball, (b) Agpeony, (c) AgFelotus (4\% Fe), (d) Ag-Fe with $10 \%$ Fe and (e) commercial PtC, (B) Nyquist plots obtained at the same electrodes (same colors and notations are used as in Fig. 3A), (C) $\mathrm{AgFe}_{\text {lotus }}$ voltammograms at various rpm (inset shows the respective Koutecky-Levich plots, arrow shows the direction of rotation speed increase) and (D) Tafel plots of (a) $\mathrm{Ag}_{\text {ball, }}$ (b) $\mathrm{Ag}_{\text {peony }}$, (c) $\mathrm{AgFe}_{\text {lotus }}$ and (d) commercial PtC electrodes. 
The superior ORR activity of $\mathrm{Ag}_{\text {peony }}$ compared to $\mathrm{Ag}_{\text {ball }}$ could be easily explained by their unique peonyflower like structures providing 8 times higher electrochemically active surface area compared to Agball (Fig. 1D and Fig.S1). On the other hand, the superior ORR activity of $\mathrm{AgFe}_{\text {lotus }}$ compared to $\mathrm{Ag}_{\text {peony }}$ could not be attributed to their higher active surface sites, since both of them expose the same electroactive surface area (Fig. S1). Thus, their respective Nyquist plots were examined to explore the role of iron particles in improving the ORR activity of silver flowers's structures (Fig. 3B). As clearly seen in Fig. 3B, Ag peony $_{\text {(curve b) displayed }}$ lower ORR charge transfer resistance than Ag ball-like particles (Agball, curve a).

Interestingly, the further modification of $\mathrm{Ag}_{\text {peony }}$ with $4 \%$ iron particles weight percent $\left(\mathrm{AgFe}_{\text {lotus, }}\right.$ curve c) led to a further decrease of ORR charge transfer resistance. That is, the iron impurities are believed to improve the silver flowers ORR catalytic activity by facilitating ORR charge transfer resistance via its $\mathrm{Fe}^{2+} / \mathrm{Fe}^{3+}$ redox couple in the same way as observed for nickel based catalyst materials ${ }^{28}$. This is indicated from the appearance of two new diffraction peaks in the $\mathrm{AgFe} \mathrm{e}_{\text {lotus }} \mathrm{XRD}$ pattern after ORR electrolysis which are attributed to $\mathrm{FeOOH}$ (see Figure S5), in addition to the presence of the $\mathrm{Fe}^{+} / \mathrm{Fe}^{3+}$ redox couple indicated from the $\mathrm{AgFe} \mathrm{e}_{\text {lotus }}$ $\mathrm{CV}$ in alkaline medium (see Fig. S6). Note that higher amounts of iron $(>4 \%)$ increase the ORR charge transfer resistance again (curve d) due to the destruction of flower-like structures. That is the higher electrocatalytic activity of $\mathrm{AgFe}_{\text {lotus }}$ flowers is attributed to their unique flowers'structures providing higher electroactive surface area and electronic interaction between silver and iron particles facilitating ORR charge transfer.

LSVs of Ag-Fe lotus flower catalysts were further recorded at various rotation rates to assess the ORR kinetics and estimate the number of transferred electrons using Koutecky-Levich plots (Fig. 3C). The average number of transferred electrons per oxygen molecule at $\mathrm{AgFe}_{\text {lotus }}$ was estimated to be $\sim 3.9$ in the whole potential window, which is similar to that of a commercial Pt electrode, indicating a favored direct 4-electron reduction pathway at our proposed Ag-Fe flower catalyst. The superb catalytic activity of AgFelotus for ORR was also demonstrated from their much smaller Tafel slope $\left(47 \mathrm{mV} \mathrm{decade}^{-1}\right)$ at low over-potentials than that measured with $\mathrm{Ag}_{\text {ball }}, \mathrm{Ag}_{\text {peony }}$ and commercial Pt/C electrocatalysts in $0.1 \mathrm{M} \mathrm{KOH}$, as shown in Figure 3D. Our studied Ag-Fe catalysts exhibited better or comparable ORR activity compared to most of the recently reported silver 
based electrocatalysts as demonstrated from its lower onset potential and Tafel slope, see comparison to other recently published data in table $\mathrm{S} 2^{8,18,21,28-32}$.

\subsection{Stability and Poisoning-Tolerance}

The catalyst durability and poisoning tolerance for various anodic fuels (by crossover) and hydrocarbon impurities are major issues for fuel cell manufacturers. Thus, the stability of the proposed Ag-Fe catalyst was further investigated and compared to that of a commercial carbon-supported Pt electrode, as presented in Fig. 4A. As seen in this figure, the $\mathrm{AgFe}_{\text {lotus }}$ catalyst (curve c) exhibited a much better stability compared to that of Agpeony (curve b) and the commercial reference (curve a). For instance, AgFe lotus activity was reduced only by $3 \%$ compared to $70 \%$ and $35 \%$ loss on the efficiency of commercial PtC and $\mathrm{Ag}_{\text {peony }}$ electrodes after 10 hours of continuous electrolysis.

The significant decrease of $\mathrm{PtC}$ activity is attributed to the aggregation/dissolution of the $\mathrm{Pt}$ active sites resulting in a significant loss of its electrochemically active surface area, as well as an increase of the ORR charge transfer resistance, as revealed in Fig. S4. This leads to the observed permanent performance loss on the commercial PtC electrode for ORR. On the other hand, Fig. 4 (B-F) and Fig. S5 show that the morphology, electrochemically active surface area and ORR charge transfer resistance of $\mathrm{AgFe}_{\text {lotus }}$ remain almost unchanged even after 7 days of continuous ORR electrolysis in alkaline medium. Additionally, the XRD pattern of AgFe lotus-like flowers after ORR electrolysis exhibited two new diffraction peaks, as shown in the inset of Fig. S5. These two diffraction peaks can be assigned to the FeOOH phase $^{33}$.

The effect of various in-situ generated HCs impurities (N.b. only the data of acetonitrile effect are presented for simplicity and the results of the other studied HCs are summarized in insets of Fig.5 and Fig. S7) from the different fuel cell components degradation on the performance of the commercial Pt reference and $\mathrm{AgFe} \mathrm{lotus}_{\mathrm{s}}$ were examined next. As revealed in Fig. S6, the presence of minute amounts ( ppm range) of these hydrocarbons' impurities such as acetonitrile, triethyl amine, vinyl acetate, and toluene resulted in a significant decrease of the commercial PtC electrode activity and stability for ORR. This performance deterioration is attributed to the deactivation of the Pt active sites by the adsorption of these HCs impurities on the Pt surface (Fig. S8) $)^{9,10}$. 

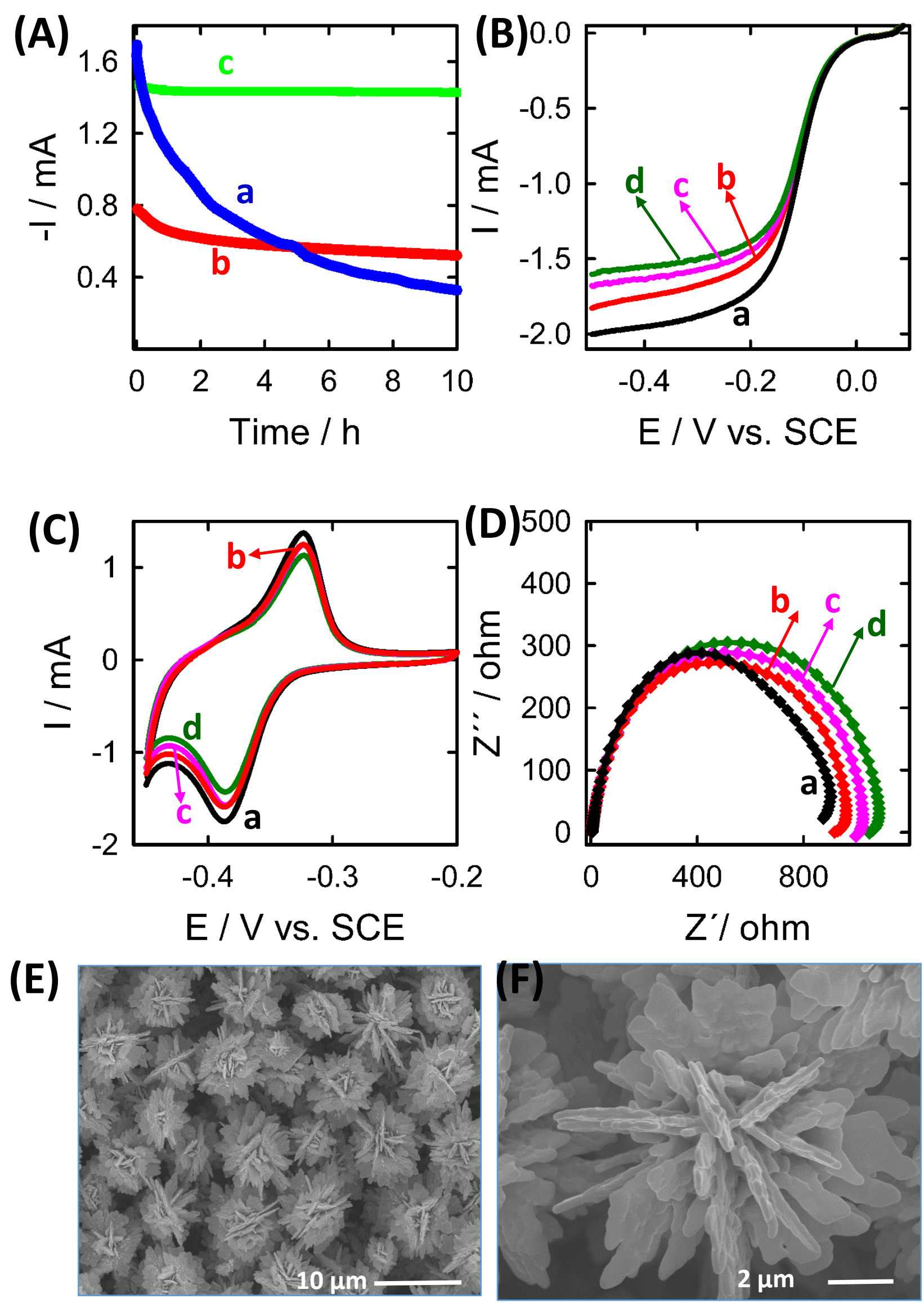

Figure 4: (A) current transient (i-t) plots obtained at (a) commercial PtC, (b) $\mathrm{Ag}_{\text {peony }}$ and (c) $\mathrm{AgFe}_{\text {lotus }}$ in $\mathrm{O}_{2^{-}}$ saturated 0.1 M KOH, (B) LSVs obtained at Ag-Fe flowers in $\mathrm{O}_{2}$-saturated $0.1 \mathrm{M} \mathrm{KOH}$ before (a) and after 2 days (b), 4 days (c) and 7 days (d) of continuous ORR electrolysis, (C) the Pb (UPD) stripping measured in $0.1 \mathrm{M} \mathrm{HCl}$ containing $10 \mathrm{mM} \mathrm{Pb}\left(\mathrm{NO}_{3}\right)_{2}$ ) and (D) Nyquist plots obtained at the same electrodes of Figure 4B (same color codes and notations are used as in Figure 4B). (E \&F) SEM images of the same electrode after 7 days of continuous ORR electrolysis with two different magnifications. 

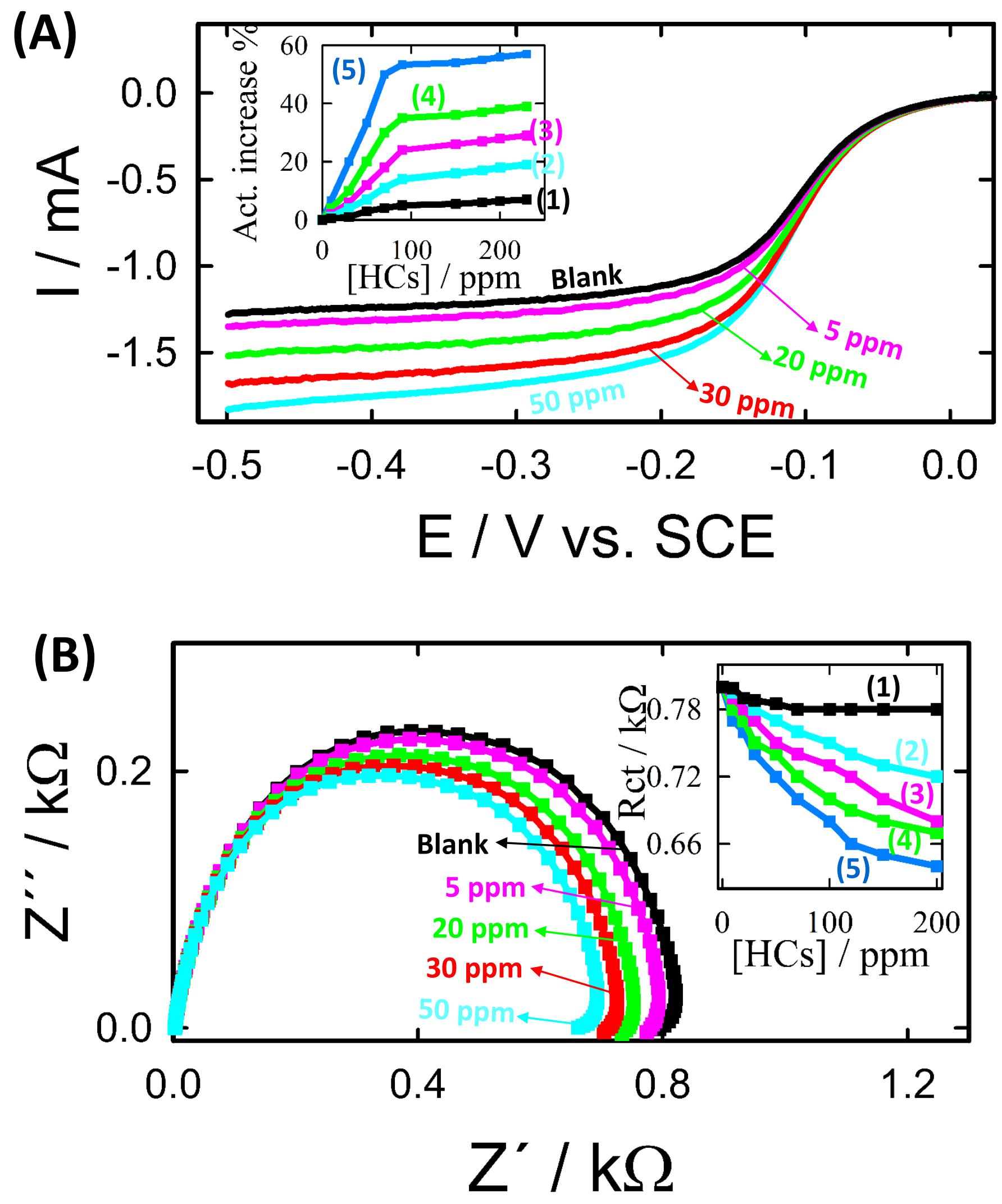

Figure 5. (A)LSVs obtained at $\mathrm{AgFe}_{\text {lotus }}$ in the presence of various acetonitrile concentrations. Insets show the variation of their respective activity with concentration of different HCs impurities (typically, acetonitrile (5), acrylonitrile (4), triethyl amine (3), vinyl acetate (2) and toluene (1)). (B) Their respective Nyquist plots in $\mathrm{O}_{2}$-saturated $0.1 \mathrm{M} \mathrm{KOH}$ containing various acetonitrile concentrations. Insets show the variation of their respective charge transfer resistance in the presence of various $\mathrm{HC}$ impurities concentrations. Note that the same electrodes, colors and notations are used as in Figure 5A. 
Interestingly, the presence of these $\mathrm{HCs}$ impurities shows no poisoning effect on the $\mathrm{AgFe}_{\text {lotus }}$ catalyst, even though they exert a slight enhancement effect for ORR (Fig. 5), as demonstrated from the slight negative shift of the ORR onset potential together with an increase of the limiting current with the increase of the hydrocarbon impurity concentration. For instance, the presence of $50 \mathrm{ppm}$ acetonitrile increases the $\mathrm{AgFe} \mathrm{lotus}_{\mathrm{s}}$ electrocatalytic activity for ORR by $55 \%$. These enhancements can be explained by the presence of these hydrocarbon impurities resulting in a small decrease of the ORR and a small reduction of the charge transfer resistance of ORR (Fig. 5). The origin of these improvements is still unclear and requires further work. However, we believe that the electronic interaction between the selected investigated hydrocarbons impurities and silver weakens the binding of the oxygen reduction intermediates ${ }^{13}$.

This is based on the fact that the decrease of ORR enhancement increases linearly with the hydrocarbon impurities dipole moments, see insets of Figure 5.

Fig. 6A shows the chronopotentiometry measurements of the commercial PtC electrode (dashed lines with empty symbols) and $\mathrm{AgFe}_{\text {lotus }}$ catalyst (solid lines with filled symbols) in $\mathrm{O} 2$-saturated $0.1 \mathrm{M} \mathrm{KOH}$ with successive additions of $50 \mathrm{ppm}$ of different HCs impurities. As clearly demonstrated from this figure, the addition of trace amounts of aforementioned HCs impurities resulted in a significant decrease of the PtC electrode performance for ORR with different extent depending on the type of HCs impurity (activity loss order: acetonitrile > acrylonitrile > triethyl amine > vinyl acetate > toluene) as demonstrated by the large negative shift of ORR onset potential with HCs addition. However, the addition of these HCs impurities slightly improved the ORR activity of $\mathrm{AgFe}_{\text {lotus }}$ electrode, indicating the higher poisoning tolerance of our suggested catalyst against different types of impurities compared to the very low tolerance of the commercial electrode.

Moreover, the tolerance of the suggested Ag-Fe catalyst towards different anodic crossover fuels (e.g., methanol, ethanol, propanol, ethylene glycol and formic acid) was examined using chronoamperometry, data are displayed in Fig. 6B. 

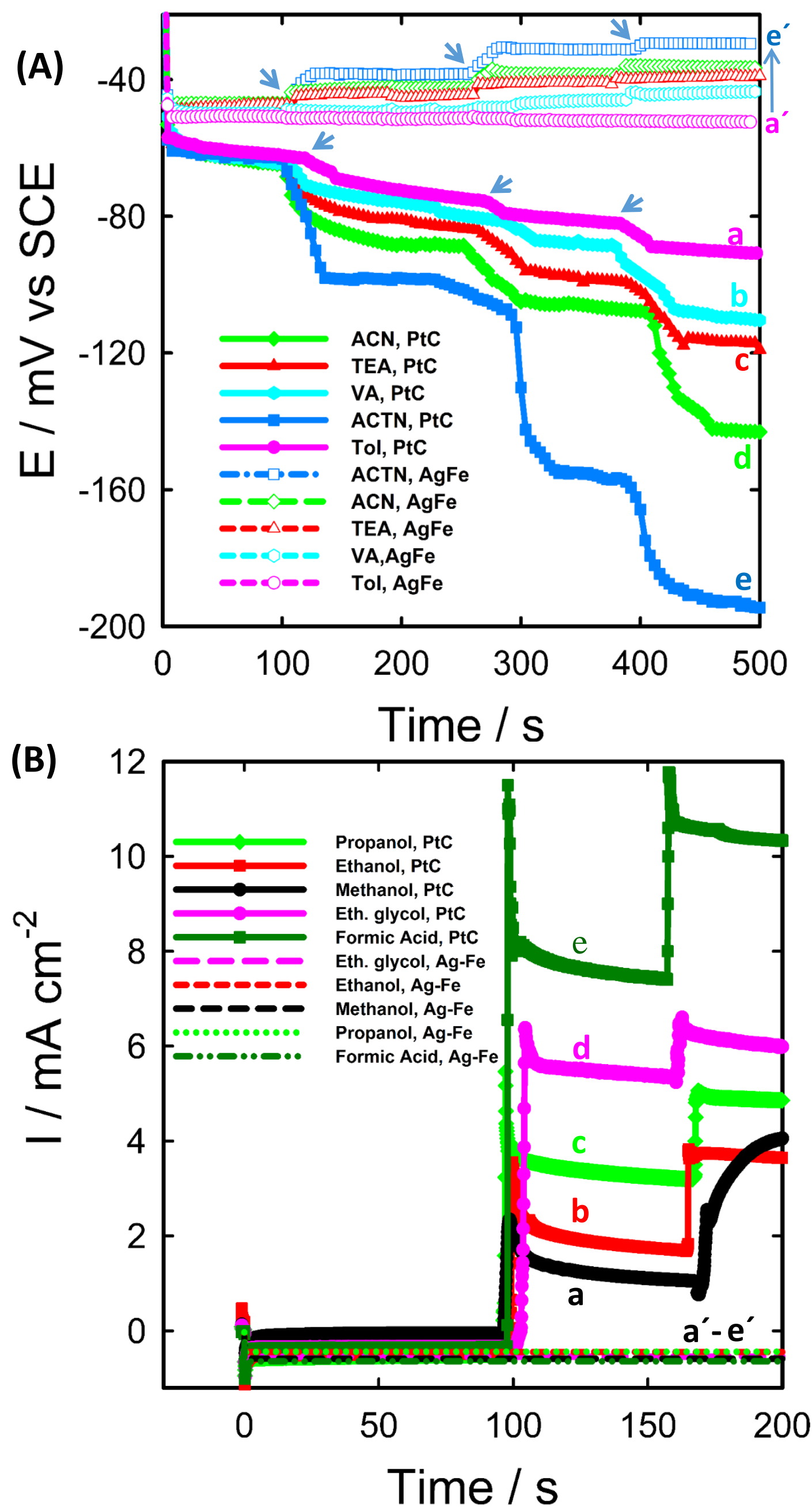
Figure 6. (A) Chronopotentiometry test of the Pt/C (filled symbols with solid lines) and Ag-Fe (empty symbols with dashed lines) electrodes at $-0.15 \mathrm{~V}$ vs. SCE during measurement successive $50 \mathrm{ppm}$ of various HCs impurities were added (arrows show the additions) (typically; toluene (pink lines, a \&a'), vinyl acetate (cyan lines, b \& b'), triethyl amine (red lines, c \& c'), acrylonitrile (green lines, $d \& d^{\prime}$ ) and acetonitrile (blue lines, e \& $\left.\mathrm{e}^{\prime}\right)$ ). (B) Chronoamperometry examination of the $\mathrm{Pt} / \mathrm{C}$ (solid lines) and $\mathrm{Ag}-\mathrm{Fe}$ (dashed lines) electrodes during measurement $1 \mathrm{M}$ of methanol (black lines, a), ethanol (red lines, b), 2-propanol (green lines, c), ethylene glycol (pink lines, d) and formic acid (dark green lines, e) were added (arrows show the additions).

As clearly shown in this figure, the addition of $1 \mathrm{M}$ of the aforementioned fuels dramatically affected the original ORR current of the commercial PtC electrode, demonstrating their oxidation, while the significant ORR activity decrease in case of formic acid is most likely attributed to CO formation from the formic acid's non-faradaic dissociation on the Pt surface to $\mathrm{CO}$ deactivating the majority of the active Pt sites. In sharp contrast, these above-mentioned fuels show no effect on the ORR chronoamperometric response of the $\mathrm{AgFe}_{\text {lotus }}$ electrode. Indeed, $\mathrm{AgFe} \mathrm{e}_{\text {lotus }}$ catalyst is an auspicious candidate for the various types of polymer electrolyte membrane fuel cells including direct alcohol fuel cells and direct formic acid fuel cells.

\section{Conclusions}

Iron-doped silver (Ag-Fe) microstructures with uniform and well-defined lotus-flower structures have been successfully prepared via a simple chemical procedure. Succinic acid served as structure directing agent to tune the roughness and morphology of the created silver particles. Silver ball-like particles were formed in the absence of succinic acid, while silver particles with peony-flower structures were created by using $2 \mathrm{mM}$ succinic acid. The as-prepared iron-doped silver lotus-flower microstructures exhibited outstanding performance as oxygen reduction reaction electrocatalyst. Ag-Fe lotus-flower catalyst exhibits a higher electrocatalytic activity, stability and poisoning tolerance for various anodic fuels crossover (e.g., methanol, ethanol and formic acid) than the commercial Pt/C catalyst. Also, it shows a higher tolerance for hydrocarbon impurities, which are in-situ generated from the degradation of various fuel cell components. This significant performance improvement is attributed to the unique flower-like structures of Ag-Fe catalyst providing a 
higher number of electroactive surface sites, besides iron particles are believed to act as catalytic mediators facilitating the ORR charge transfer resistance.

\section{Acknowledgements}

This work was financially supported by Alexander von Humboldt Foundation (AvH). Gumaa A. El-Nagar is grateful for a fellowship from the AvH. 


\section{References}

1. S. Cherevko, N. Kulyk and K. J. J. Mayrhofer, Nano Energy, 2016, 29, 275-298.

2. X. Fu, F. M. Hassan, P. Zamani, G. Jiang, D. C. Higgins, J.-Y. Choi, X. Wang, P. Xu, Y. Liu and Z. Chen, Nano Energy, 2017, 42, 249-256.

3. L. Du, Y. Shao, J. Sun, G. Yin, J. Liu and Y. Wang, Nano Energy, 2016, 29, 314-322.

4. M. Arenz and A. Zana, Nano Energy, 2016, 29, 299-313.

5. G. A. El-Nagar, M. A. Hassan, I. Lauermann and C. Roth, Scientific Reports, 2017, 7, 17818.

6. J. Liang, Y. Jiao, M. Jaroniec and S. Z. Qiao, Angewandte Chemie International Edition, 2012, 51, 1149611500.

7. J. Liang, Y. Zheng, J. Chen, J. Liu, D. Hulicova-Jurcakova, M. Jaroniec and S. Z. Qiao, Angewandte Chemie International Edition, 2012, 51, 3892-3896.

8. H. Lee, M. J. Kim, T. Lim, Y.-E. Sung, H.-J. Kim, H.-N. Lee, O. J. Kwon and Y.-H. Cho, Scientific Reports, 2017, 7, 5396.

9. M. S. El-Deab, F. Kitamura and T. Ohsaka, Journal of Power Sources, 2013, 229, 65-71.

10. M. S. El-Deab, F. Kitamura and T. Ohsaka, Journal of The Electrochemical Society, 2013, 160, F651-F658.

11. G. A. El-Nagar, A. M. Mohammad, M. S. El-Deab and B. E. El-Anadouli, ACS Applied Materials \& Interfaces, 2017, 9, 19766-19772.

12. G. A. El-Nagar, R. M. Sarhan, A. Abouserie, N. Maticiuc, M. Bargheer, I. Lauermann and C. Roth, Scientific Reports, 2017, 7, 12181.

13. G. A. El-Nagar, M. S. El-Deab, A. M. Mohammad and B. E. El-Anadouli, Electrochimica Acta, 2015, 180, 268279.

14. G. A. El-Nagar, A. M. Mohammad, M. S. El-Deab, T. Ohsaka and B. E. El-Anadouli, Journal of Power Sources, 2014, 265, 57-61.

15. K. I. Ozoemena, RSC Advances, 2016, 6, 89523-89550.

16. D. R. Dekel, Journal of Power Sources, 2018, 375, 158-169.

17. Y.-J. Wang, J. Qiao, R. Baker and J. Zhang, Chemical Society Reviews, 2013, 42, 5768-5787.

18. R. Zhou and S. Z. Qiao, Chemistry of Materials, 2014, 26, 5868-5873.

19. P. Singh and D. A. Buttry, The Journal of Physical Chemistry C, 2012, 116, 10656-10663.

20. B. B. Blizanac, P. N. Ross and N. M. Markovic, Electrochimica Acta, 2007, 52, 2264-2271.

21. A. Holewinski, J.-C. Idrobo and S. Linic, Nature Chemistry, 2014, 6, 828.

22. X. Sun and M. Hagner, Langmuir, 2007, 23, 9147-9150.

23. L. Wang, H. Li, J. Tian and X. Sun, ACS Applied Materials \& Interfaces, 2010, 2, 2987-2991.

24. R. J. Chimentao, I. Kirm, F. Medina, X. Rodriguez, Y. Cesteros, P. Salagre and J. E. Sueiras, Chemical Communications, 2004, DOI: 10.1039/B400762J, 846-847.

25. K. Kim and J. W. Han, Physical Chemistry Chemical Physics, 2016, 18, 27775-27783.

26. T. T. Nhung and S.-W. Lee, ACS Applied Materials \& Interfaces, 2014, 6, 21335-21345.

27. X.-L. Liu, J.-H. Wang, S. Liang, D.-J. Yang, F. Nan, S.-J. Ding, L. Zhou, Z.-H. Hao and Q.-Q. Wang, The Journal of Physical Chemistry C, 2014, 118, 9659-9664.

28. H. A. Miller, M. Bevilacqua, J. Filippi, A. Lavacchi, A. Marchionni, M. Marelli, S. Moneti, W. Oberhauser, E. Vesselli, M. Innocenti and F. Vizza, Journal of Materials Chemistry A, 2013, 1, 13337-13347.

29. N. Zhang, F. Chen, X. Wu, Q. Wang, A. Qaseem and Z. Xia, Journal of Materials Chemistry A, 2017, 5, 70437054.

30. A. Qaseem, F. Chen, X. Wu, N. Zhang and Z. Xia, Journal of Power Sources, 2017, 370, 1-13.

31. Z. Lin and X. Qiao, Scientific Reports, 2018, 8, 1802.

32. S. Li, H. Miao, Q. Xu, Y. Xue, S. Sun, Q. Wang and Z. Liu, RSC Advances, 2016, 6, 99179-99183.

33. J. Wang, L. Li, C. L. Wong, L. Sun, Z. Shen and S. Madhavi, RSC Advances, 2013, 3, 15316-15326. 